\title{
Anticoagulation during ECMO: Will the Tight Rope be Tighter in 2018?
}

\author{
Poonam Malhotra Kapoor ${ }^{1}$ \\ 1Department of Cardiac Anaesthesiology, Cardio Thoracic Centre, \\ All India Institute of Medical Sciences, New Delhi, India
}

\section{J Card Crit Care TSS 2017;1:55-56}

Extracorporeal membrane oxygenation (ECMO), the machine we love to hate, is a lifesaving modality today in every medical/surgical intensive care unit (ICU). The biggest obstacle to increasing survival with ECMO is a triad of neurological injury, hematologic injury, and organ recovery ${ }^{1}$ ( Fig. 1A and B).

The largest organ in the body is not skin or liver, but it is the endothelium, which in the ECMO foreign surface, undergoes endothelial disruption, and is the major cause of bleeding in ECMO. There is activation of platelets and all other factors involving the foam cells, nitric oxide pathway, endothelin, and other factors following platelet adhesion and aggregation which leads to hemolysis following ECMO. ${ }^{2}$

\begin{abstract}
Address for correspondence Poonam Malhotra Kapoor, MD, DNB, MNAMS, FIACTA (Hony), FTEE (Hony), FISCU (Hony), Department of Cardiac Anaesthesiology, Cardio Thoracic Centre, All India Institute of Medical Sciences, New Delhi, India (e-mail: docpoonamaiims@gmail.com).
\end{abstract}

It is surprising that despite 40 years of struggle with ECMO components, designs, and circuitry, this lifesaving machinery is still not available in each medical/surgical ICU and not adopted universally as well. Anticoagulation studies on ECMO ( - Fig. 1) though on the rise in the last 10 years do not still give definitive information on which is the right anticoagulation, even though heparin has been used by all so far.

Anticoagulants, thus, are needed during ECMO which would inhibit platelets and prevent coagulation activation within the ECMO circuit. The complications of most anticoagulants including heparin are bleeding, thrombosis, heparin resistance, and heparin-induced thrombocytopenia. Heparin

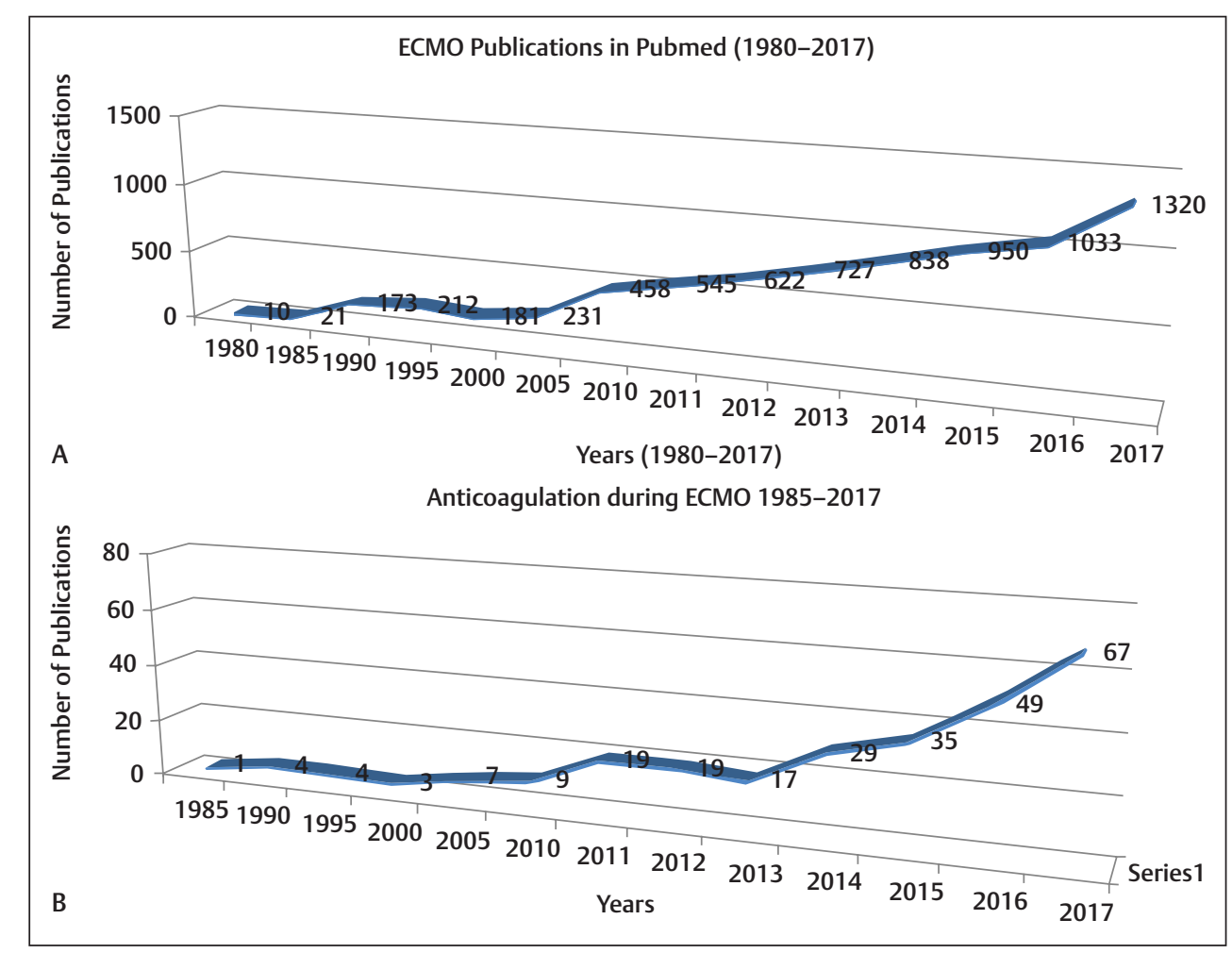

Fig. 1 (A) Extracorporeal membrane oxygenation (ECMO) publications in PubMed 1980-2017. (B) Anticoagulation during ECMO publications in PubMed 1985-2017.

DOI https://doi.org/

$10.1055 / \mathrm{s}-0038-1626680$

ISSN 2457-0206.
Copyright $\odot 2017$ Official Publication of The Simulation Society (TSS), accredited by International Society of Cardiovascular Ultrasound (ISCU)
License terms

()ㅇㅇ $\Theta \circledast$ 
is not a direct thrombin inhibitor! It acts by activating antithrombin III. Monitoring antithrombin III is essential in ECMO, as low levels of ATIII lead to clot formation despite high doses of heparin (heparin resistance). Also, heparin is responsible for platelet aggregation and adhesion. Heparin so far used in ECMO is dependent on antithrombin III for its activity. Bivalirudin or argatroban, both direct thrombin inhibitors, may be heparin alternatives for ECMO, thus making anticoagulation simpler and easier to titrate. ${ }^{3}$ Thus, studies on heparin alternatives continue and so do the indigenous ways of monitoring heparin activity.

Sanfilippo et al studied bivalirudin and explained it to be the best viable option for patients on ECMO who present contraindications to or ineffectiveness of heparin anticoagulation. Bivalirudin has been used both with and without a loading dose, and as is seen in heparin anticoagulation, bivalirudin also requires strict and continuous dose adjustment according to prespecified targets. Prospective larger randomized controlled studies are necessary to fully determine both the safety and efficacy for the use of bivalirudin during ECMO. ${ }^{3}$

Point of care testing with thromboelastography and/or rotational thromboelastometry with platelet aggregometry with arachidonic and adenophosphate inhibitors for monitoring are futuristic trends that will pave the way for existing limitations of the assessment of hemostasis in adult and pediatric ECMO patients. Reliable information on hypofibrinogenemia with hyperfibrinolysis during ECMO with "maximum amplitude" as the most sensitive predictor of diagnosing bleeding within 15 minutes of sampling is here to stay and so is TRAPTEM/ADPTEM/ARATEM on the ROTEM platelet aggregometry monitor studies which need to be extensively undertaken as multicentric larger cohorts. ${ }^{4,5}$

The dreaded complication of bleeding during ECMO is due to the disruption of the endothelium, when exposed to an artificial medium leads due to platelet adhesion and aggregation. What is needed today are artificial ECMO devices, which will have surfaces that will inhibit platelet adhesion to this artificial surface. All this will decrease the need for artificial anticoagulation during ECMO. ${ }^{2}$ Some such platelet adhesion inhibiting material are nitric oxide like substances along with intramembranous lungs are futuristic trends to prevent ECMO bleeding.

This July-December 2017 issue of JCCC is home to special manuscripts on ECMO: Articles addressing development of the ECMO technique; growing trends of ECMO in Indian subcontinent; use of ECMO in poisoning patients with aluminum phosphide for toxicology in ICU, unique in India; ROTEM values on a VA ECMO case showing fibrinogen deficiency; immunological aspects in organ transplant in India; and update on extracorporeal circulatory support highlighting different aspects and significance of ECMO. Bleeding can be prevented in this ECMO circuitry with new molecules incorporated and faster point of care testing to make a right blood product choice at the right time. ECMO is lifesaving machinery and there is hope in this therapy, what is needed is research in multicentric trials with larger cohorts! The name of Baby Esperanza, the first ECMO survivor, still means hope as we find new circuits, miniature components, novel ways to anticoagulate, ventilate, and cannulate these patients! The HOPE for ideal anticoagulation during ECMO, continues.

If any reader of this ECMO special issue would like to be in touch with me, please email: e-mail: edmjccc@gmail.com.

\section{References}

1 Daneshmand MA, Milano CA. ECMO: 40 years later. ASAIO J 2017;63(6):693-694

2 Bartlett RH. ECMO: the next ten years. Egypt J Crit Care Med 2016;4:7-10

3 Sanfilippo F, Asmussen S, Maybauer DM, et al. Bivalirudin for alternative anticoagulation in extracorporeal membrane oxygenation: a systematic review. J Intensive Care Med 2017;32(5):312-319

4 Bhardwaj V, Malhotra P, Hasija S, Chowdury UK, Pangasa N. Coagulopathies in cyanotic cardiac patients: an analysis with three point - of - care testing devices (Thromboelastography, rotational thromboelastometry, and sonoclot analyzer). Ann Card Anaesth 2017;20(2):212-218

5 Petricevic M, Konosic S, Biocina B, et al. Bleeding risk assessment in patients undergoing elective cardiac surgery using $\operatorname{ROTEM}(\circledR)$ platelet and Multiplate $(\circledR)$ impedance aggregometry. Anaesthesia 2016;71(6):636-647 